\title{
Why benzodiazepines are not going
} away $^{\dagger}$

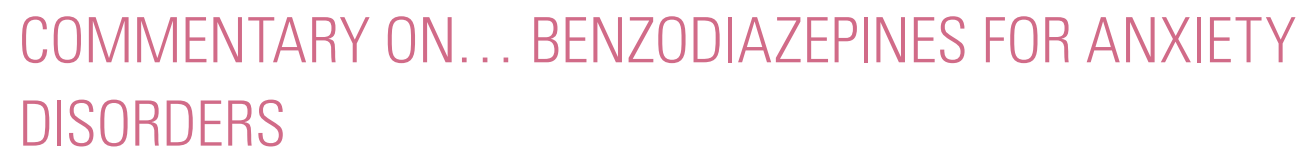

\section{Peter Tyrer}

\begin{abstract}
SUMMARY
Benzodiazepines are still widely prescribed in psychiatry, mainly for the treatment of anxietyrelated disorders. This is not because they are only taken by a hard core component of patients who are addicted and do not need them; it is because they remain the most effective and rapid acting of all drug treatments. Strategies for practitioners to use them more confidently are described.
\end{abstract}

\section{DECLARATION OF INTEREST}

None.

This is an overdue article on the place of benzodiazepines in current psychiatric practice (Starcevic 2012, this issue). Despite all the concerns about their risks in the past 30 years, all attempts to reduce prescriptions have failed, and some deplore this state of affairs (Lader 2011). Yet the important message often forgotten is that their prescription is not increasing, and this was the main alarm 40 years ago, when there were even suggestions that these drugs could be made available to people without prescription. Starcevic is also right to point out that the primary concern expressed by both doctors and patients about the use of benzodiazepines is related to their alleged addiction potential. What follows in this commentary are some observations that may appear to be 'off field' in differing from many guidelines but which I feel are based on good evidence and, more importantly, reflect the problems patients and their practitioners face in practice, and these are not always appreciated by armchair commentators.

Four areas of concern need to be addressed if benzodiazepines are to be prescribed appropriately and, although these are stressed to some extent in Starcevic's article, there are some differences which are relevant for a UK audience. It is first important to say that the main concerns about benzodiazepines have been expressed in the UK and Australia, whereas in other countries, especially France, but also the USA, the worries about the prescription of benzodiazepines have been much less.

\section{Addiction potential of benzodiazepines}

Starcevic is certainly right to point out that a lot of the concern over the addiction potential of benzodiazepines was fostered by pharmaceutical firms that were introducing new antidepressants, particularly the selective serotonin reuptake inhibitors (SSRIs), in the 1980s. When many of these showed some evidence of efficacy in anxiety disorders, there was a natural consequence to both recommend and prescribe SSRIs for all the anxiety disorders for which benzodiazepines were formerly prescribed (Tyrer 2006). Nevertheless, despite many guidelines recommending this change, practitioners have stuck stubbornly to benzodiazepines (Blanco 2004).

There is also a clear distinction between high-dose and low-dose dependence. High-dose dependence is found with drug misusers who take benzodiazepines in excessive dosage, very often with other drugs of dependence, and benzodiazepines in such dosage have been associated with significant problems in the past, particularly with temazepam, which was extracted from its capsule and injected (Forsyth 1993). But only a small minority of patients taking benzodiazepines are high-dose misusers. Almost all the others are taking the drugs for anxiety disorders and when they develop dependence it is only after continuous prescription of relatively low doses. Low-dose dependence is not associated with an escalation of dosage and there is relatively little evidence of tolerance, but it can, on cessation, lead to a withdrawal syndrome (Tyrer 1980; Petursson 1981; Ashton 1984). It is sometimes not fully appreciated that withdrawal problems occur in only approximately $40 \%$ of those who take benzodiazepines regularly (Tyrer 1981).

There are continuing arguments over differences in addiction potential between benzodiazepines but
Peter Tyrer is Professor of Community Psychiatry at the Department of Psychological Medicine, Imperial College London, UK. Correspondence Professor Peter Tyrer, Imperial College London, Department of Medicine, Centre for Mental Health, Charing Cross Campus, St Dunstan's Road, London W6 8RP, UK. Email: p.tyrer@ imperial.ac.uk

${ }^{\dagger}$ See pp. 250-258, this issue. 
no unequivocal conclusions can be drawn from the data (Burrows 1990). Nevertheless, there is strong circumstantial evidence that benzodiazepines that have high potency and/or short elimination half-lives (e.g. triazolam, alprazolam, lorazepam) provoke more withdrawal problems than lowpotency drugs with longer elimination halflives (chlordiazepoxide, nitrazepam), although it should be borne in mind that some shortacting benzodiazepines have long-acting active metabolites (Tyrer 1993). What is interesting is that the same pattern of withdrawal symptoms has been found with SSRIs, although these can be associated with many other symptoms as well (Price 2009). It is also relevant that SSRIs of shorter elimination half-life are associated with more withdrawal problems than those of longer half-life, although other factors are also relevant here (Baldwin 2007).

Although it is common for pharmaceutical companies to refer to these withdrawal symptoms as a 'discontinuation syndrome', this is mere semantics. The simple fact is that withdrawal syndromes occur with both antidepressants and sedative antianxiety drugs, and we should not pretend that they are not withdrawal phenomena and so linked to dependence. Yet there are differences. The most interesting difference between benzodiazepines and antidepressants (and indeed many other anti-anxiety drugs) is that the calming effect of the sedative agents is associated with a degree of pleasure, probably linked to their facilitation of GABA transmission, also found with alcohol. Other drugs, such as buspirone, have calming effects but are associated with a degree of dysphoria, and this explains why they have never been favoured much in clinical practice, even though they are free from withdrawal problems (Murphy 1989). It is possible to associate the pleasure that some people get from taking benzodiazepines with the euphoria of dependence, but it is not really the same when patients are taking these drugs in relatively low doses.

However one looks at it, the withdrawal problems that patients often manifest when they stop taking benzodiazepines are a matter of concern because not only can they lead to long-term prescription that might be unnecessary but also because withdrawal symptoms can be prolonged.

\section{Alternatives}

Although the SSRIs were heavily promoted as alternatives to the benzodiazepines, they have never really replaced them, for the reasons that Starcevic suggests. The rapid onset of action of benzodiazepines makes them much more appropriate for sudden episodes of anxiety (including panic but not always such severe anxiety) and explains why they are preferred. What are now commonly called the Z-drugs (zaleplon, zolpidem, zopiclone) have been promoted as alternatives to benzodiazepines, but it is very important for practitioners to realise that these drugs also attach to benzodiazepine binding sites even though there are some differences in their pharmacological actions (Wilson 2010). It is also relevant that the selection of anxiety or insomnia for a drug treatment is mainly dependent on dose - and if the Z-drugs were given regularly during the day for anxiety disorders (they are all marketed as hypnotics) they might well lead to the same withdrawal symptoms as benzodiazepines. Although there is a lack of studies in this area, one recent report claims to show no evidence of dependence resulting from prolonged treatment with zolpidem, but this needs to be replicated (Roehrs 2011).

\section{Duration of treatment}

Where current guidelines differ from practice is that the prescription of benzodiazepines is almost universally recommended to be short term, and no longer than 4 weeks. In practice, people like taking benzodiazepines because they relieve anxiety, but most forms of anxiety are not resolved within 4 weeks, so it is not surprising that a majority of patients go on taking them for much longer periods. In fact, when these drugs are taken for 6 weeks and then tailed off gradually, the outcome at 10 weeks is significantly worse than taking placebo (Tyrer 1988), and I am far from certain that the extra 2 weeks beyond 4 weeks is critical here. My judgement now, and it has changed somewhat since 1980 , is that standard guidelines that recommend taking these drugs in regular dosage for no longer than 4 weeks are making two wrong assumptions: first, that all patients are liable to dependence if they continue regular dosage beyond 4 weeks, and second, that their anxiety problem will have resolved by the time they stop medication.

In my own work I have always considered two strategies, which I put to the patient, as I think a degree of patient choice here is relevant. The first strategy is to avoid dependence at all costs. I explain to the patient that benzodiazepines have a strong tendency to induce dependence and the best way to prevent this is to take them only when needed, and preferably not on more than 3 days a week. This policy carries the advantages that when the drug is taken it has an immediate and dramatic effect, as drug-induced tolerance is low, and that the taking of a tablet is regarded as a special event, not a regular drudge. The second 
strategy is to explain the risks of dependence and the possibility of this happening if dosage is taken long term, but to explain this in detail and to ask the patient to come to a personal decision. As my policy is always to assess whether the patient has any personality problems, my choice of strategy is often determined by the absence or presence of dependent personality characteristics, and so I specify this association in my advice. In practice, I find that a significant minority of patients choose to take a small dose of a benzodiazepine long term and without any obvious problems ensuing. Although there has been concern over long-term cognitive impairment with chronic benzodiazepine use, this remains to be proved and for some people the consumption of low-dose benzodiazepines is regarded as the best solution for chronic anxiety, a view that has been reflected in at least some consensus statements (Uhlenhuth 1999). This strategy appears to condone dependence, but it is one which has been recognised as appropriate for many patients whose quality of life would otherwise be terrible.

\section{Choice of patient}

Although Starcevic suggests that patients with panic disorder are most appropriate to treat with benzodiazepines, there is so much overlap between the different anxiety disorders that it is often difficult to tell which condition a drug is actually being given for. What we have found in previous work (Tyrer 1983), but it needs to be confirmed by others, is that those with anxious dependent personalities, and possibly those with obsessional ones too, are more likely to develop dependence on benzodiazepines than those of other personality types. If we could make a good assessment of personality at the beginning of treatment, we might be able to select those who could take benzodiazepines and those for whom they really should be avoided. We are already making progress in this direction and a short questionnaire, the Dependent Personality Questionnaire (Tyrer 2004), could be used to screen those who are at risk of dependence even before starting therapy. It is a self-rating questionnaire that takes $5 \mathrm{~min}$ to complete, is a reliable measure of dependent personality features as assessed by interview and is also relatively stable over time (Tyrer 2012). We are gradually moving towards a better selection procedure for those who might need long-term benzodiazepines, and this is sorely needed in a risk-averse world.

Over 20 years ago, in a highly charged article, the greatly respected clinical psychiatrist Dr Frederick Kräupl Taylor castigated those who wished to abandon the use of the benzodiazepines (Taylor 1989). He ended this with words that still have resonance today:

'Unfortunately, the increasingly strident and threatening clamour against the therapeutic use of benzodiazepines compels doctors today to be unusually circumspect, and to temper their medicinal advice with a (recorded) caution of the side-effects patients might experience in the first few days (e.g. somnolence, clumsiness, perhaps an aggressive outburst), and of the possibility of a traumatic morbid dependence on prolonged medication. One cannot expect the general public to have much respect and therapeutic concern for patients with an anxiety illness. Yet they are in special need of our sympathy and understanding for the stigma they endure and the wretchedness they suffer, which can drive some of these unfortunates to seek a despairing exit through suicide.'

\section{References}

Ashton H (1984) Benzodiazepine withdrawal: an unfinished story. BMJ 288: 1135-40.

Baldwin DS, Montgomery SA, Nil R, et al (2007) Discontinuation symptoms in depression and anxiety disorders. International Journal of Neuropsychopharmacology 10: 73-84.

Blanco C, Goodwin RD, Liebowitz MR, et al (2004) Use of psychotropic medications for patients with office visits who receive a diagnosis of panic disorder. Medical Care 42: 1242-6.

Burrows GD, Norman TR, Judd F, et al (1990) Short-acting versus longacting benzodiazepines: discontinuation effects in panic disorders. Journal of Psychiatric Research 24 (suppl 2): 65-72.

Forsyth AJ, Farquhar D, Gemmell M, et al (1993) The dual use of opioids and temazepam by drug injectors in Glasgow (Scotland). Drug and Alcohol Dependence 32: 277-80.

Lader M (2011) Benzodiazepines revisited - will we ever learn? Addiction 106: 2086-109.

Murphy SM, Owen R, Tyrer P (1989) Comparative assessment of efficacy and withdrawal symptoms after 6 and 12 weeks' treatment with diazepam or buspirone. British Journal of Psychiatry 154: 529-34.

Petursson H, Lader MH (1981) Withdrawal from long-term benzodiazepine treatment. BMJ 283: 643-5.

Price J, Cole V, Goodwin GM (2009) Emotional side-effects of selective serotonin reuptake inhibitors: qualitative study. British Journal of Psychiatry 195: 211-7.

Roehrs TA, Randall TA, Harris E, et al (2011) Twelve months of nightly zolpidem does not lead to rebound insomnia or withdrawal symptoms: a prospective placebo-controlled study. Journal of Psychopharmacology Oct 16 (epub ahead of print).

Starcevic V (2012) Benzodiazepines for anxiety disorders: maximising the benefits and minimising the risks. Advances in Psychiatric Treatment 18: 250-258.

Taylor FK (1989) The damnation of benzodiazepines. British Journal of Psychiatry 154: 697-704

Tyrer P (1980) Dependence on benzodiazepines. British Journal of Psychiatry 137: 576-77.

Tyrer P (1993) Pharmacological differences between benzodiazepines. In Benzodiazepine Dependence (ed C Hallstrom): 221-37. Oxford University Press.

Tyrer P, Baldwin D (2006) Generalised anxiety disorder. Lancet 368 : 2156-66.

Tyrer P, Rutherford D, Huggett T (1981) Benzodiazepine withdrawal symptoms and propranolol. Lancet 317: 520-2.

Tyrer P, Owen R, Dawling S (1983) Gradual withdrawal of diazepam after long-term therapy. Lancet 321: 1402-6. 
Tyrer P, Seivewright N, Murphy S, et al (1988) The Nottingham study of neurotic disorder: comparison of drug and psychological treatments. Lancet 332: 235-40.

Tyrer P, Morgan J, Cicchetti D (2004) The Dependent Personality Questionnaire (DPO): a screening instrument for dependent personality. International Journal of Social Psychiatry 50: 10-7.

Tyrer H, Tyrer P, Barrett B (2012) Influence of dependent personality on the outcome and service costs of health anxiety. International Journal of Social Psychiatry Mar 19 (epub ahead of print).
Uhlenhuth EH, Balter MB, Ban TA, et al (1999) International study of expert judgment on therapeutic use of benzodiazepines and other psychotherapeutic medications: IV. Therapeutic dose dependence and abuse liability of benzodiazepines in the long-term treatment of anxiety disorders. Journal of Clinical Psychopharmacology 19 (suppl 2): 23S-9S.

Wilson SJ, Nutt DJ, Alford C, et al (2010) British Association for Psychopharmacology consensus statement on evidence-based treatment of insomnia, parasomnias and circadian rhythm disorders. Journal of Psychopharmacology 24: 1577-601.

\title{
IN OTHER WORDS
}

\section{'It was the youth of Dr. Howell': extract from Faces in the Water, by Janet Frame}

\author{
Selected by Femi Oyebode
}

\begin{abstract}
Janet Frame (1924-2004) was born in Dunedin, New Zealand. Reportedly, only the award of a literary prize for a collection of short stories saved her from leucotomy and prompted her release from an 8-year confinement in a psychiatric hospital. Frame drew on her experiences in mental institutions in the novel Faces in the Water, which set in New Zealand between the First and Second World Wars. This extract is from Faces in the Water, The Women's Press, 1980: pp. 29-30.
\end{abstract}

doi: 10.1192/apt.18.4.262
It was the youth of Dr. Howell which appealed to us; the other doctors who did not look after us but who were in charge of the hospital were gray-haired and elderly and hurried in and out of their offices down in front of the building like rats in and out of their hiding places; and they sat, in their work, with the same old chewed solutions littered about them, like nesting material. It was Dr. Howell who tried to spread the interesting news that mental patients were people and therefore might like occasionally to engage in the activities of people. Thus were born "The Evenings" when we played cards - snap, old maid, donkey and euchre; and ludo and snakes and ladders, with prizes awarded and supper afterwards. But where was the extra staff to supervise the activities? Pavlova, the one Social Worker for the entire hospital, valiantly attended a few "social" evenings held for men and women patients in the Ward Four dayroom. She watched people mount ladders and slide down chutes and travel home on the red and blue squares of parcheesi. She too was pleased when the climax of the evening came with the arrival of Dr. Howell in sport coat and soft shoes, with his corn-colored hair slicked down and his undoctorly laugh sounding loud and full. He was like a god; he joined in the games and threw the dice with the aplomb of a god hurling a thunderbolt; he put in the appropriate expression of dismay when he was ordered to slide down a chute, but you could see that he was a charmer even of bilegreen cardboard snakes. And of people. He was Pavlova's god too, we knew that; but no amount of leaping about in her soiled white coat with the few bottom buttons undone could help her to steal Dr. Howell from the occupational therapist. Poor Pavlova! And Poor Noeline, who was waiting for Dr. Howell to propose to her although the only words he had even spoken to her were How are you? Do you know where you are? Do you know why you are here? - phrases which ordinarily would be hard to interpret as evidence of affection. But when you are sick you find yourself in a new field of perception where you make a harvest of interpretations which then provides you with your daily bread, your only food. So that when Dr. Howell finally married the occupational therapist, Noeline was taken to the disturbed ward. She could not understand why the doctor did not need her more than anyone else in the world, why he had betrayed her to marry someone whose only virtue seemed to be the ability to show patients who were not always interested, how to weave scarves and make shadow stitch on muslin. 